\title{
NEVERS, Prieuré Saint-Etienne
}

René de LESPINASSE (éd.), « Les chartes de Saint-Etienne de Nevers », in Bulletin de la Société nivernaise des Lettres, Sciences et Arts, 22 (1908), p. 51-130.

\section{Marie-José Gasse-Grandjean}

\section{(2) OpenEdition Journals}

Édition électronique

URL : https://journals.openedition.org/cem/5512

DOI : $10.4000 /$ cem. 5512

ISSN : 1954-3093

\section{Éditeur}

Centre d'études médiévales Saint-Germain d'Auxerre

\section{Référence électronique}

Marie-José Gasse-Grandjean, « NEVERS, Prieuré Saint-Etienne », Bulletin du centre d'études médiévales d'Auxerre / BUCEMA [En ligne], Collection CBMA, mis en ligne le 05 septembre 2008, consulté le 24 septembre 2022. URL : http://journals.openedition.org/cem/5512 ; DOI : https://doi.org/10.4000/cem. 5512

Ce document a été généré automatiquement le 24 septembre 2022.

\section{(c) (i) (9)}

Creative Commons - Attribution - Pas d'Utilisation Commerciale - Partage dans les Mêmes Conditions 4.0 International - CC BY-NC-SA 4.0

https://creativecommons.org/licenses/by-nc-sa/4.0/ 


\section{NEVERS, Prieuré Saint-Etienne}

René de LESPINASSE (éd.), « Les chartes de Saint-Etienne de Nevers », in Bulletin de la Société nivernaise des Lettres, Sciences et Arts, 22 (1908), p. 51-130.

\section{Marie-José Gasse-Grandjean}

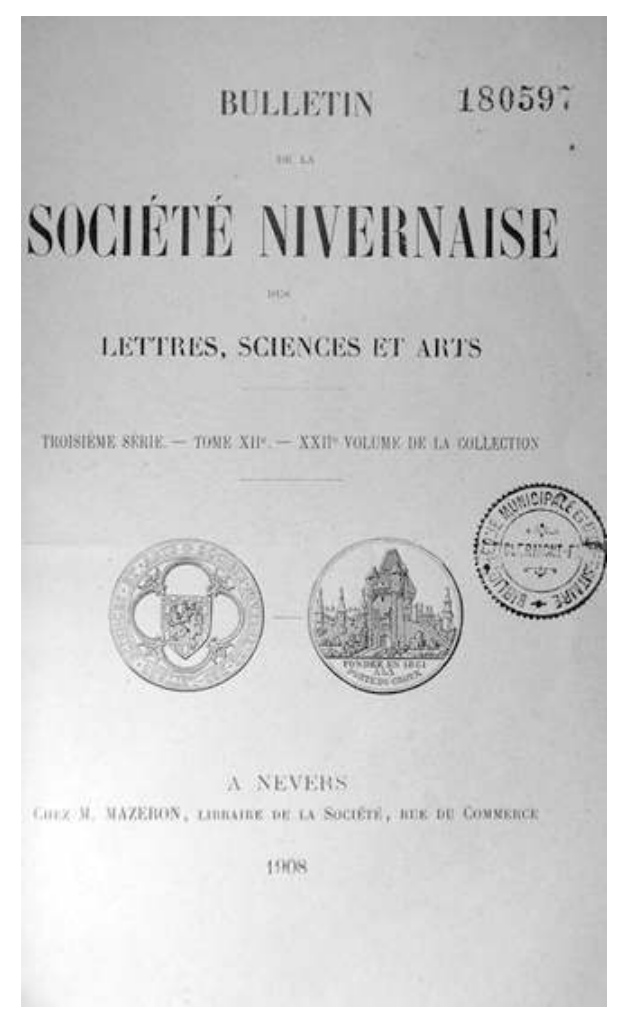

\section{Historique}

Saint-Etienne (et Notre-Dame) de Nevers serait une fondation colombanienne du début du VIIe siècle. Communauté de femmes ruinée au IXe siècle, rénovée au XIe siècle par l'évêque pour y établir des chanoines mais sans grand succès, elle fut finalement offerte à l'abbaye de Cluny en 1068. Cluny y fonda un prieuré bénédictin dépendant en 1097 et cette date est celle de la première charte éditée par René de Lespinasse. Les comtes de 
Nevers dotèrent largement cette nouvelle fondation. Autour de l'abbaye, le faubourg Saint-Etienne placé sous l'autorité du prieur bénéficia d'un statut particulier. L'abbatiale romane consacrée le 13 décembre 1097, aujourd'hui église paroissiale magnifiquement conservée ${ }^{1}$, témoigne encore du prestige souhaité par les comtes pour cet établissement.

Archives et documentation

Dans l'inventaire des cartulaires bourguignons établi récemment ${ }^{2}$, les cartulaires nivernais répertoriés sont peu nombreux: sept unités documentaires dont deux cartulaires de l'évêché et de Saint-Cyr, trois cartulaires de Saint-Etienne, un "bullaire " de Saint-Germain d'Auxerre concernant le prieuré Saint-Pierre de Decize et un cartulaire de l'abbaye Saint-Pierre-le-Moutier. Parmi ces sept cartulaires, quatre sont perdus, deux sont des fragments. La documentation apparait donc très modeste et l'espace nivernais très sous-représenté dans cet inventaire bourguignon. Un fragment de cartulaire (C) et un recueil de privilèges (D) concernent le prieuré Saint-Etienne.

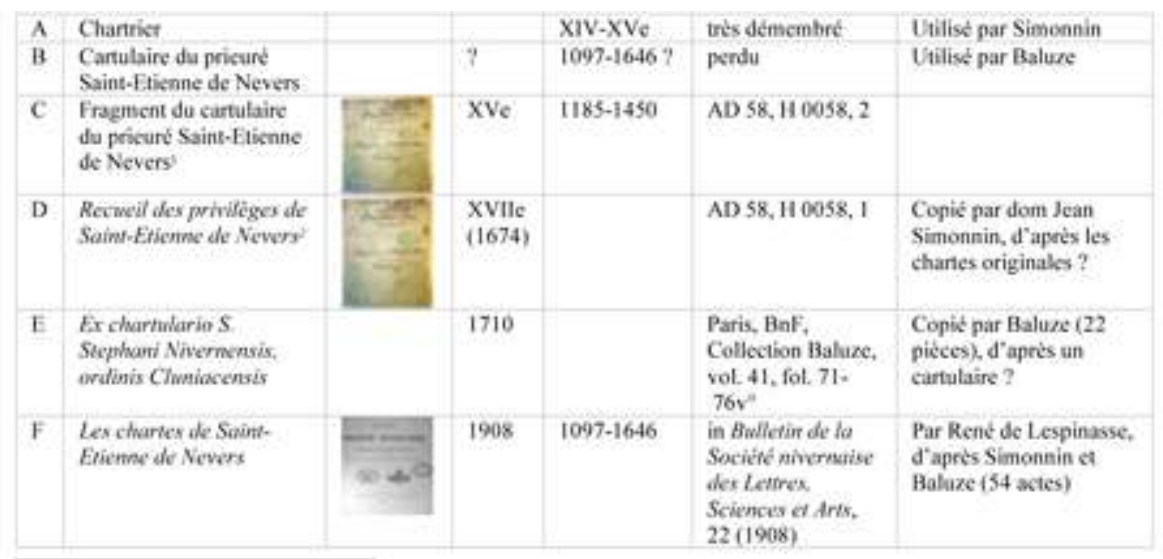

' Ce manuscrit sur parchemin, au format presque earré ( $170 \times 180 \mathrm{mn})$, rélié par un simple cordon, réunit dix actes bien indivodualises sur six folios.

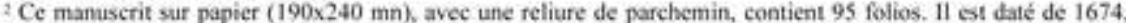
signé par Jean Simonnin et dédié à Henri Bertrand de Beuvron, nouvel abbé de Cluny. C'est un recueil soigné. classé et annoté.

\section{La documentation de Saint-Etienne}

L'éditionL'éditeur Louis-René Leblanc de Lespinasse (1843-1922)

R. de Lespinasse a consacré une grande part de ses recherches à l'histoire nivernaise, dans le cadre de la Société nivernaise des Lettres, Sciences et Arts dont il fut le président pendant de longues années. Il publia au final une monumentale histoire des comtes de Nevers ${ }^{3}$. Chartiste ${ }^{4}$, il fut aussi un éditeur de documents diplomatiques ${ }^{5}$.

Contenu de l'édition

4 Une introduction de 25 pages précède l'édition des actes. Elle concerne davantage les actes que la méthode d'édition. R. de Lespinasse signale simplement qu'il a classé les chartes " par ordre de dates, soit in extenso, par extraits ou par simple cote, selon leur intérêt » et qu'il en exposera "les divers objets qu'elles traitent » (p. 57). Après une présentation historiographique courte, il évoque tour à tour chaque charte ou presque, situant l'action juridique, traduisant parfois une partie du texte, identifiant certains personnages. On regrette cependant que des renvois vers cette documentation ne soient pas faits depuis/vers l'édition des actes ${ }^{6}$.

5 R. de Lespinasse a édité chronologiquement 54 actes diplomatiques dont les dates s'échelonnent entre 1097 et 1646, 41 actes étant antérieurs à 1300. Il fait un choix 
d'actes ${ }^{7}$ et donne la priorité aux actes inédits. Après l'acte de fondation du prieuré, il privilégie quantitativement les actes comtaux (14), les bulles pontificales (11), les actes épiscopaux (8), les diplômes royaux et actes du Parlement (6), et les actes des prieurs (5). Viennent ensuite les actes de particuliers qui ratifient ou bien des donations ou bien des ventes au prieuré.

Qualité de l'édition

6 R. de Lespinasse a travaillé à partir d'actes diplomatiques, de terriers et d'anciens manuscrits. Mais a t-il enquêté systématiquement dans les archives et les bibliothèques? On peut s'étonner par exemple qu'il ne donne pas le texte de la charte d'Hugues, évêque de Nevers, restaurant l'abbaye Saint-Etienne de Nevers en 1063, conservée en original dans la Collection de Bourgogne à Paris (BnF, t. 78, $\left.\mathrm{n}^{\circ} 114\right)$ et éditée par Bernard et Bruel dans leur recueil des chartes de Cluny (Paris, Imprimerie nationale, 1888 , t. 4 , p. 487-490, $\mathrm{n}^{\circ}$ 3388). R. de Lespinasse renvoie simplement à cette édition en note 1 dans l'introduction, et signale, au total, seulement cinq actes originaux conservés.

7 Il est difficile ensuite de savoir ce que R. de Lespinasse a consulté effectivement. A t-il consulté le fragment de cartulaire du XVe siècle? Aux archives départementales de la Nièvre par exemple, il n'a pas collationné les actes originaux conservés dans la liasse Privilèges $\mathrm{H}$ 59. Quand il donne l'édition de l'acte de serment de l'évêque Jean d'Etampes (29 mai 1446, acte 49), il reproduit les fautes de transcription de Jean Simonnin (prouti au lieu de prout uti, manum tenere au lieu de manutenere, Abiron pour Abyron...). Mais l'original était-il disponible alors? La question se pose quand on constate que l'archiviste Henri de Flamare (1882-1911) a transcrit le même texte d'après le cartulaire du XVe siècle, renvoie à Simonnin mais ne fait jamais allusion à l'original conservé.

8 R. de Lespinasse a utilisé les outils locaux et généraux disponibles (Gallia christiana, Collection Grangier de la Marinière...) et principalement deux éditions d'actes diplomatiques antérieures, celle de Baluze $^{8}(1710)$ et celle de dom Jean Simonnin ${ }^{9}$ (1674). R. de Lespinasse indique ces éditions dès le titre de son travail : Chartes de SaintEtienne d'après Baluze et Dom Simonnin. Sur 54 numéros d'actes, 28 ont édités d'après Simonnin, 11 d'après Baluze, 10 d'après Simonnin et Baluze. Il décrit les éditions qu'il a utilisées : il signale ainsi qu'on y trouve une préface, des dessins de bulles et de monogrammes, des notes marginales, des mentions de sceaux ou une liste des prieurs; il indique "sans date » ou bien "in extenso »; il corrige parfois, et s'interroge sur l'existence d'un cartulaire ancien à partir de l'indication de Baluze « ex chartulario $\mathrm{S}$. Stephani Nivernensis ordinis Cluniacensis ${ }^{10}$.

9 R. de Lespinasse adopte un classement chronologique des actes. Mais il propose rarement de nouvelles dates, de nouvelles identifications ou de nouvelles lectures, et il argumente peu ses choix. Il mêle analyses et transcriptions, titres et analyses. Il abrège préambules et textes, et choisit par exemple de ne pas reproduire certaines formules jugées " ordinaires». Il référence mal ces textes. Il cite l'édition de Simonnin par exemple sans rigueur, on trouve Recueil de Dom Simonnin..., Recueil Simonnin..., Simonnin..., Copiée par dom Simonnin... Parfois il omet tout à fait d'indiquer une citation ${ }^{11}$. Ou bien il signale un acte conservé aux "Archives départementales de la Nièvre " sans plus de précision (acte 48). Ou bien il oublie de citer sa source (actes 30, 31 et 50). 


\section{BIBLIOGRAPHIE}

CROSNIER Augustin-Joseph, «L'église et le prieuré de Saint-Etienne de Nevers », in Bulletin de la Société nivernaise des Lettres, Sciences et Arts, 4 (1853), p. 108-178.

LESPINASSE René de, Le Nivernais et les comtes de Nevers, 3 vol., Paris, 1909-1914.

ZENNER Marie-Thérèse, « Methods and meaning of physical analysis in romanesque architecture : a case of study, Saint-Etienne in Nevers », Ph. D. dissertation, Bryn Mawr College, 1994.

ZENNER Marie-Thérèse, « Saint-Etienne de Nevers : un ancien prieuré de Cluny dans le Nivernais », in Les Annales des pays nivernais, 80 (1995).

CHAGNY Anne-Marie, « Saint-Etienne de Nevers. Les sources aux archives départementales », in Semaine religieuse du diocèse de Nevers, 21 (1997), p. 503-507.

\section{NOTES}

1. Christian Sapin, La Bourgogne romane, avec la collaboration de Chantal Arnaud et Walter Berry, Dijon, 2006, p. 232-235.

2. Isabelle Rosé, « Panorama de l'écrit diplomatique en Bourgogne autour des cartulaires (XI ${ }^{\mathrm{e}}$-XVIII ${ }^{\mathrm{e}}$ siècles)», in Bulletin du Centre d'études médiévales d'Auxerre, 11 (2007), mis en ligne le 29 juin 2007. URL : http://cem.revues.org/document1535.html.

3. Le Nivernais et les comtes de Nevers, 3 vol., Paris, 1909-1914.

4. Essai sur deux comtes de Nevers Hervé de Donzy, Guy de Forez, Mahaut de Courtenay, comtes et comtesse de Nevers de 1199 à 1257, thèse de l'Ecole des chartes, 1867.

5. Outre les chartes de Saint-Etienne et de Saint-Cyr de Nevers, il publia le Cartulaire du prieuré de La Charité-sur-Loire (Paris, 1887), les Chartes nivernaises du comte de Chastellux (Nevers, 1896-[1898]), Les plus anciennes chartes du Nivernais jusqu'au Xe siècle (in Bulletin de la Société Nivernaise des Lettres, Sciences et Arts, 9 (1902), p. 229-245) et les Titres du prieuré de Mèves-sur-Loire, XIIe-XVIIIe s. (idem, 7 (1899), p. 171-232).

6. Page 70-71, il rédige un développement au sujet du droit de merallus donnant un exemple parisien et soulignant la singularité du terme dans le dictionnaire de Ducange. Page 89, quand il édite l'acte par lequel le comte Guillaume accorde au prieur de Saint-

Bulletin du centre d'études médiévales d'Auxerre | BUCEMA, Collection CBMA 
Etienne l'autorisation d'avoir un merallus, il ne fait aucune référence aux explications précédentes.

7. Lespinasse ne reprend pas par exemple une bulle de Clément VI, conservée et éditée par Simonnin (p. 108-113), ou les actes signalés par l'abbé de Marolles dans son Inventaire des titres de Nevers... (Nevers, 1873, tome 1, p. 489 seq.).

8. Paris, BnF, Coll. Baluze, ms. 41, fol. $71-76 v^{\circ}$. Baluze y réunit des extraits d'actes diplomatiques et complète avec une liste des prieurs comme dom Simonnin.

9. Procureur du prieuré de Saint-Etienne, dom Jean Simonnin a travaillé d'après les actes diplomatiques originaux et d'anciens manuscrits, et composé un Recueil des titres de Saint-Etienne de Nevers, conservé aujourd'hui aux Archives départementales de la Nièvre (H 0058,1). Il a signalé aussi « les terriers du prieuré », mais il ne semble en avoir utilisé qu'un seul, sans autre précision (p. 126).

10. Baluze attribue à quelques actes des numéros en chiffres romains (VII, VIII, XII, XIIII, XXI) qui peuvent rappeler une numérotation ancienne. Aucune autre indication matérielle ne permet cependant de retenir l'hypothèse d'un cartulaire constitué.

11. Page 126, il ajoute, à la suite de l'acte 52 daté de 1472, le paragraphe suivant : "Semblable accord fust passé la même année pour le lict de feu damoiselle Jehanne de Veaulce, femme de noble homme Helyon de Druy, et plusieurs autres de diverses années se trouvent dans les terriers du prieuré que nous n'avons peu inserer à cause de leur longueur et multiplicité, nous estans restreinct à ce dernier qui suit pour preuve et marque fidelle de la conservation dudict droict ». Les tournures et l'orthographe de l'ancien français permettent de comprendre que ce texte est un commentaire de Jean Simonnin, repris par Lespinasse sans aucun avertissement.

12. Au sujet des juifs par exemple, «toujours habiles à profiter des circonstances », p. 66.

INDEX

Index géographique : France/Nevers

Mots-clés : charte bourguignonne 